\title{
Le fou dans le théâtre espagnol des années 1920, une figure de crise
}

David Coste

\section{(2) OpenEdition}

1 Journals

Édition électronique

URL : https://journals.openedition.org/cher/3882

DOI : $10.4000 /$ cher.3882

ISSN : 2803-5992

Éditeur

Presses universitaires de Strasbourg

\section{Édition imprimée}

Date de publication : 1 décembre 2015

Pagination : 221-230

ISBN : 978-2-86820-913-9

ISSN : 1968-035X

\section{Référence électronique}

David Coste, «Le fou dans le théâtre espagnol des années 1920, une figure de crise », reCHERches [En ligne], 15 | 2015, mis en ligne le 01 décembre 2021, consulté le 15 décembre 2021. URL : http:// journals.openedition.org/cher/3882 ; DOI : https://doi.org/10.4000/cher.3882

\section{(c) (i) (8)}

Ce(tte) œuvre est mise à disposition selon les termes de la Licence Creative Commons Attribution Pas d'Utilisation Commerciale - Partage dans les Mêmes Conditions 4.0 International. 


\title{
Le fou dans le théâtre espagnol des années 1920, une figure de crise
}

\author{
DAVID COSTE \\ Université de Strasbourg - EA4376-C.H.E.R.
}

\begin{abstract}
$\mathrm{L}$ e théâtre des années 1920 voit naître en Espagne la veine d'un théâtre psychanalytique, dit aussi théâtre freudien qui met en scène un nouveau personnage à la croisée des représentations du fou théâtral traditionnel, inspiré des fêtes de carnaval, et du dément interné. La médicalisation de la folie dans ces pièces s'inspire des théories freudiennes et rend compte d'un psychisme qui a cessé dêtre envisagé comme un tout homogène et cohérent. Le conflit psychique qui oppose la conscience aux désirs inconscients fait de ce personnage de fou une figure de crise en soi, en tant que représentant sur scène du dysfonctionnement d'un être victime de ses contradictions intimes. Cette communication se propose d'interroger cette figure et ce théâtre freudien comme "théâtre critique" ou théâtre de la crise en regard du concept de "poétique de la crise» mis en lumière notamment par Sylvain Diaz dans sa récente thèse sur les poétiques de la crise dans les dramaturgies européennes des Xxe et XxI ${ }^{\mathrm{e}}$ siècles (Diaz: 2009).

Le terme crise nous rappelle S. Diaz vient étymologiquement, dans la langue grecque, du terme de krisis qui désigne dans les domaines juridique, médical et théologique un même "jugement subjectif émis au terme de délibérations approfondies» (Diaz 2009: 15). Sous l'effet notamment de la théorisation médicale du terme de crise par Hippocrate, la crise prend un sens temporel et signifie «l'instant décisif», le moment du jugement dans l'évolution du malade ou d'un processus, le moment où «ça se décide». Tour à tour investie dans le domaine politique, économique et social, à partir du XIX ${ }^{e}$ siècle, la notion de crise devient fonctionnelle dans le champ poétique et entre dans les traités d'esthétique théâtrale. La crise devient un concept interprétatif de la tragédie, notamment de la tragédie classique.

Concept polysémique, dans la langue courante, la crise indique alors une phase grave dans l'évolution d'une situation, un moment paroxystique qui met à jour le dysfonctionnement d'un corps ou d'un système.
\end{abstract}


C'est au regard de cette polysémie que je me propose d'interroger l'existence d'un théâtre de la crise dans le théâtre psychanalytique espagnol des années 1920 .

Ce théâtre psychanalytique est d'emblée situé thématiquement dans le champ d'un «théâtre critique» dans la mesure où il représente les dérèglements psychiques d'un homme faisant face aux vertiges de sa conscience. La brèche que le freudisme a ouverte dans le monolithisme de la personnalité conduite par la conscience se dit dramatiquement par la représentation d'un homme en quête de soi. Le doute a envahi la pensée et les certitudes élémentaires sont mises à mal dans des dramaturgies qui, à la sortie de la seconde guerre mondiale, exploitent ces théories pour en tirer des représentations de l'homme tantôt hilarantes, tantôt angoissantes.

Les fous de l'asile de Sinrazón de Sánchez Mejías incarnent des hommes et des femmes qui souffrent d'une profonde crise identitaire. L'homme en crise est un homme qui s'est inventé une nouvelle identité et que les représentants de la médecine psychiatrique tentent de ramener sur le chemin de la vérité. La conscience de soi est minée par la chimère, par le délire construit dramatiquement par la peinture de l'incomplétude qui façonne ces êtres en quête de vérité sur leur Moi profond. Tous les fous de Sinrazón sont marqués au début de la pièce par le sceau de la misère. Les didascalies insistent sur les haillons que portent les fous et sur l'image misérable qu'ils renvoient: «Van llegando locos atropelladamente. Visten como vagabundos, con ropa sucia y harapienta» (Sánchez Mejías 1988: 65), «La reina viste pobre y estrafalariamente» (Sánchez Mejías 1988: 67). L'image de cet avatar de reine dégradée, misérable mais reine tout de même, renvoie à l'ampleur de la crise identitaire des fous de l'asile.

La représentation visuelle de la crise se loge dans cet écart incommensurable entre le délire de personnalité de l'homme en quête de son Moi et la réalité asilaire plus que décevante. Ainsi, la réalité psychique du fou construit un monde cohérent et structuré qui contraste littéralement avec l’aspect visuel du personnage et l'image est celle, dissonante et quelque peu absurde, d'un fou pathétique.

La crise de folie, entendue comme un accès de violence subite ou comme l'expression d'angoisses profondes constitue l'un des motifs du dérèglement psychique dans ces pièces. Ce théâtre psychanalytique dépeint une humanité déchaînée qui est sortie de ses gonds. À l'image critique de l'homme en quête de sa vérité profonde s'ajoute un aspect plus temporel de la crise entendue comme instant paroxystique d'une tension extrême.

L'incarnation comique de cette figure de crise est illustrée dans de nombreuses pièces à travers la récurrence du fou furieux au théâtre. Dans Tararí de Valentín Andrés Álvarez, le fou furieux apparaît au tout début du premier acte, à travers les paroles du Loco $1^{\circ}$ : «[...] llegará al Sanatorio un loco furioso» (Andrés Álvarez 1929: 11). Le fou furieux est annoncé en son absence, préparant ainsi, aux deux niveaux de l'énonciation théâtrale, la crise qu'il va jouer. Deux scènes plus tard, le fou furieux entre en scène à mi-chemin entre le portrait du fauve et 
celui du surhomme. L'image scénique va forcer le trait spectaculaire de la crise en surjouant le renversement inattendu qui sopère. La crise dramatique, préparée en amont peut alors avoir lieu. Amené comme un fauve dont le moindre geste peut être fatal, la didascalie souligne toute la surenchère qui préside à l'image scénique: «[...] cuando ve venir al Portero, al Loquero $1^{\circ} \mathrm{y}$ al Vigilante, los cuales traen al Loco $2^{\circ}$, sujetándolo cada uno por un brazo. El portero traerá colgada de un hombro la camisa de fuerza. El Loco $2^{\circ}$ forcejea con lo que le llevan, tirando hacia atrás» (Andrés Álvarez 1929: 16). Trois hommes sont nécessaires pour en maintenir un qui se débat. Laccent est mis sur le lieu commun de la dangerosité $\mathrm{du}$ fou. Visuellement, la raison s'impose par le nombre mais le fou parvient à mettre à terre ses geôliers sans aucun mal comme l'indique la didascalie qui suit: «De dos golpes derriba a dos vigilantes. Lucha con los demás y se apodera de la camisa de fuerza que tiene en sus manos el Portero» (Andrés Álvarez 1929: 17). Tout cela participe à l'élaboration de l'image d'un fou furieux, à la force spectaculaire, qui confine à la monstruosité. L'homme en crise dans cette farce comique est convoqué ici en tant que véritable ressort de l'intensité dramatique. La crise du fou furieux est ici l'instant paroxystique du point de vue de l'intensité dramatique mais aussi l'instant décisif, au regard de l'économie générale de la pièce. Après la crise, plus rien ne sera comme avant et la fable se noue ici dans une intensité liée au ressort spectaculaire que constitue l'homme déchaîné sur scène. Plus qu'un sujet critique, la «crise de folie» est un instrument qui désigne un temps dramaturgique précis: celui du renversement.

Pour autant, les manifestations de la crise dans ce théâtre freudien ne s'arrêtent pas au portrait d'un homme en crise. À cela s'ajoute, notamment dans les pièces comiques, un dérèglement politique qui révèle une crise à l'échelle collective cette fois.

Dans Tararí, autour de la crise spectaculaire du fou furieux s'articule un renversement politique des rôles au sein de l'asile puisque les internés prennent le pouvoir pour imposer aux personnes sensées leur vision des choses. Cette prise de pouvoir des fous est explicite puisque la pièce, à partir de ce moment clef qu'est la troisième scène du premier acte, file la métaphore de la lutte armée et du putsch militaire. Les didascalies pointent la dimension militaire du renversement à travers la figure du chef: "Erigiéndose en general en jefe» (Andrés Álvarez 1929: 18). Puis: «Todos siguen al Loco $2^{\circ}$ como a un caudillo» à la fin de la scène. Ils forment une petite armée. La camisole de force vient compléter ce tableau de la lutte armée, étant érigée comme létendard d'un nouvel ordre: celui de la folie: «El Loco $2^{\circ}$ sostiene en alto la camisa de fuerza como un estandarte» (Andrés Álvarez 1929: 17). La crise du fou furieux au premier acte est donc pleinement une crise en ce sens qu'elle est non seulement un moment paroxystique du point de vue de la tension dramatique de cette pièce mais aussi parce qu'elle constitue un «instant décisif» qui produit un renversement de l'intrigue. Autour de la crise individuelle, qui passe finalement au second plan, sarticule une crise collective qui remet en question la société dans son ensemble. Le fou est une figure de 
crise politique en ce sens qu'il est le porte-parole d'un fonctionnement social radicalement différent. Il est la voix qui porte et initie une autre vision du monde.

On retrouve le même schéma dans Sinrazón où la crise de folie criminelle de Don Manuel assassinant le docteur Ballina renvoie à un dérèglement collectif. Là encore, la crise apparaît comme un instant décisif: celui où disparaît sur scène, et donc dans ce microcosme sociétal, le dernier représentant de la raison scientifique. La crise inaugure ainsi un temps où la folie est maîtresse de son destin. La crise est, là encore, individuelle et collective. Le meurtre du docteur qui clôt la pièce symbolise un temps irréversible, à savoir : l'instant où se décide l'avenir d'un homme retombé, à travers l'assassinat, dans la folie la plus sombre, ainsi que l'instant où se cristallise l'avenir d'une communauté livrée dès lors à elle-même.

Thématiquement donc le théâtre psychanalytique fait de la crise psychique un sujet dramatique. Dans la veine d'un théatre qui cherche un rire facile, sous la plume de Muñoz Seca ou de Carlos Arniches, la représentation de l'homme en crise sert de coup de théâtre dont la fonction dramatique est de créer la situation comique. L'homme en crise apparaît comme une manne de théâtralité que s'approprient ces dramaturges pour transmettre des propos tout à fait conventionnels et codifiés, dans la lignée des thématiques de vaudeville. Dans la Locura de Don Juan, l'homme en crise cache un mari soumis tandis que dans La Cura, la crise feinte d'Óscar et de Fontillonga sert de couverture à ces deux maris volages. Même la crise véritable de Genoveva est symptomatique du carcan social de la bourgeoisie madrilène, fait de conventions qui ne laissent aucune place au divertissement gratuit. La figure de l'homme en crise est invoquée dans ces dramaturgies parce qu'elle est spectaculaire et donc pourvoyeuse de mille ressorts d'équivoques comiques.

En revanche, la crise apparaît au sens dramaturgique plein «d'instant décisif», de "retournement irréversible» dans des pièces comme Tararí ou Sinrazón pour signifier l'articulation qui sétablit entre une personnalité troublée et un changement politique radical.

Néanmoins la seule thématique critique ne permet pas de conclure hâtivement à l'existence d'un théâtre de crise.

La crise se construit également d'un point de vue esthétique. La thèse de Sylvain Diaz insiste, à partir de la conception médicale de la crise chez Hippocrate, sur une crise au théâtre qui ne saurait être comprise comme un moment dramaturgique mais plutôt comme un processus, une opération de mise en crise la distinguant ainsi de la catastrophe tragique. Cette conception dramaturgique de la crise comme processus la rend extérieure au drame qu'elle envisage dans sa totalité, dans sa globalité. La crise est une interprétation du drame, une vision du drame: "Toute crise est opération critique. Toute crise est d'abord et avant tout mise en crise» (Diaz 2009: 37). C'est cette idée de «mise en crise», comme processus esthétique qu'il s'agit maintenant de confronter au théâtre freudien espagnol des années 1920. 
D’un point de vue spatial, tout d'abord, comment les espaces scéniques de ces pièces sont-ils pensés comme des lieux de crise? Ce théâtre, en dépit de sa modernité, applique la règle aristotélicienne des trois unités qui étaient, dès la Poétique, la garante d'un théâtre où peut avoir lieu la crise tragique. Dès le théâtre tragique grec, la crise s'incarne dans une esthétique de la concentration extrême qui assure cette surtension dramatique dans laquelle elle sépanouit. Sans tension dramatique, aucune crise n'est possible. Dans ces conditions, «l'espace resserré» de l'unité spatiale est sans surprise l'option suivie par ces auteurs espagnols pour planter le décor de la crise. Mais l'unité de lieu n'est pas suffisante pour faire surgir la crise. Tous ces dramaturges ont fait le choix d'un espace clos pour faire éclater la crise. Tararí et Sinrazón mettent en scène des fous de l'asile. Lespace clos, l'emprisonnement dit le resserrement nécessaire au surgissement de cette surtension. Dans les deux cas, l'espace scénique est certes un espace extérieur mais les frontières de l'asile n'en sont pas plus prégnantes dans l'esprit des internés.

La symbolique de l'espace de la crise est encore plus claire dans les pièces aux couleurs d'avant-garde d'Azorín et de Rivas Cherif. Le troisième volet de la trilogie, Doctor Death de 3 a 5, d’Azorín est significatif quant à la fonction que revêt l'espace scénique dans la structuration de la crise. La pièce met en scène l'attente d'une jeune malade qui vient consulter le bien nommé docteur Death pour guérir de son mal-être existentiel. Ici l'espace scénique représenté est la salle d'attente de la consultation du docteur Death. De nouveau, l'enfermement physique va de pair avec le surgissement de la crise. La surtension critique est ici le résultat de la construction de l'espace scénique comme espace de nonretour. Une fois que la protagoniste entre dans la salle d'attente, elle ne peut plus en échapper. Lauteur insiste sur cet espace du confinement qui dès le début est difficile d'accès:

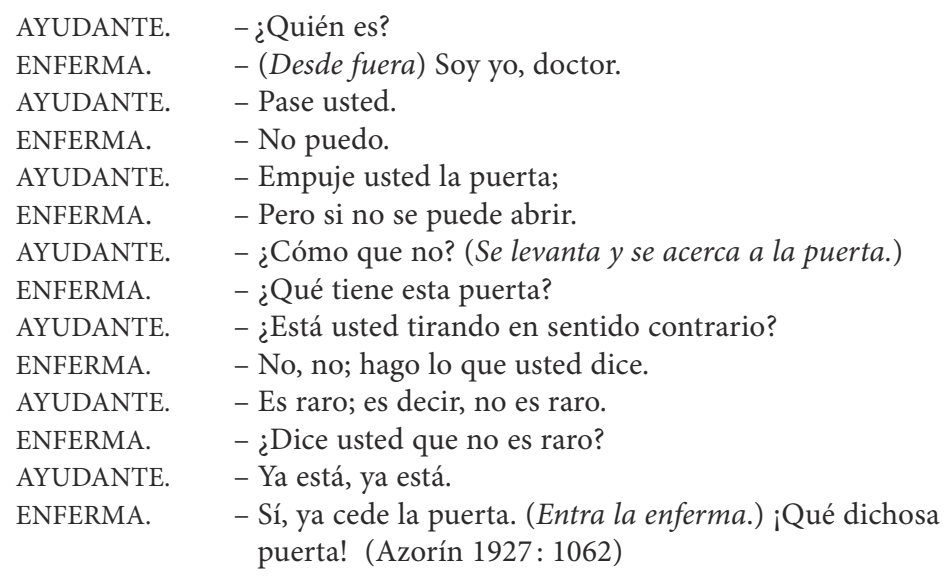

Après avoir eu des difficultés pour rentrer dans la salle d'attente, l'espace scénique devient sans surprise un d'espace d'enfermement dont elle ne pourra plus sortir: "(Se acerca rápidamente a la puerta y trata de abrirla.) No se puede abrir. La abriré con mis uñas, con toda mi persona. Quiero abrirla... No se puede. ¡No 
es posible abrir la puerta, no es posible escapar!» (Azorín 1927: 1069). Lespace scénique est dès lors d'autant plus critique qu'elle est la seule ne pas pouvoir en sortir. Celle qui n'a d'autre nom que «la enferma» est d'abord abandonnée par «el ayudante del doctor» avant de lêtre par «el Viejo». La salle d’attente devient un lieu de solitude, un mouroir où la crise d'angoisse va naître. L'espace critique du confinement a pris chez Azorín la forme d'une intériorité, d'un inconscient extériorisé qui se projette sur l'espace scénique. Cette salle d'attente, point de non-retour et antichambre de la mort, n'est autre que la dimension plastique du conflit psychique qui ronge la protagoniste. Le blocage, le dysfonctionnement psychique se donne dans un espace scénique symbolique qui préfigure la crise.

Dans Trance de Rivas Cherif la crise fait une fois de plus appel à la représentation d'un espace intérieur. L'espace retenu par le dramaturge est celui du foyer du docteur où une patiente vient soigner son somnambulisme par une séance d'hypnose. Lespace intimiste de la maison n'est pas ici emprisonnant mais il conforte l'idée d'un espace critique à l'abri des regards. Le lieu de la crise va dans cette pièce d'autant plus se soustraire au regard d'autrui quau début de la séance d'hypnose, le thérapeute éteint la lumière: "Deme usted las manos. Siéntese usted cómoda. ¿Le molesta a usted la luz? Sí, voy a apagarla...» (Rivas Cherif 1923: 3). Lorsque les lumières éclairent de nouveau l'espace scénique, à l'issue de la séance, le médecin gît mort sur son siège, victime de la crise meurtrière de sa patiente: “¿Dónde estás? Enciende la luz, ¡anda!... Es muy tarde... Bueno, iré yo... Profesor... Prof... ¿Eh..? ¿Qué es...esto...? ¡Aaaaaah...!!!! Aaaaaasesinooo!» (Rivas Cherif 1923: 6)

Qu'il soit le lieu d'un enfermement ou un intérieur intimiste, l'espace critique, se construit comme un espace d'exception, coupé du reste du monde. C'est en ce sens qu'il permet le surgissement de la tension nécessaire à la situation de crise.

Cependant, l'espace du confinement ne saurait qu'en partie construire la tension dramatique indispensable à l'éclatement de la crise. L’unité de temps est déjà pointée du doigt par Aristote comme pourvoyeuse de tension dramatique. L'histoire doit se déployer dans un temps restreint pour qu'il y ait crise. Pour autant, toutes les dramaturgies qui respectent l'unité de temps ne correspondent pas à ce "théâtre de crise». Comment se construit alors cet "instant décisif» qu'est la crise?

D'un point de vue temporel, la crise se caractérise par la notion de rupture violente. La crise surgit sans prévenir. Si la crise peut avoir des prémices, il ne saurait y avoir crise sans surprise, sans intensification brutale de la tension dramatique, sans accélération du rythme de l'action dramatique. Plusieurs des pièces de ce corpus s'ouvrent sur un temps d'oisiveté, de calme imperturbable qui permet ensuite d'initier la rupture rythmique de la crise. C'est le cas de la première scène de Tararí qui souvre sur une scène placée sous le signe de l'apaisement. L'espace scénique représente le jardin de l'asile. La didascalie indique la présence d'un gardien qui lit un journal. Cette activité précise le calme qui règne dans un univers sous contrôle, maîtrisé, apaisé. La première image scénique de la pièce de Doctor Death de 3 a 5 s'inscrit dans cette même idée d'un 
temps de crise qui se construit par contrastes. Lassistant du docteur y apparaît en train de lire paisiblement.

Dans La Cura, la didascalie qui ouvre la pièce va dans le même sens. L'espace du premier acte est le jardin d'un hôpital psychiatrique qualifié comme tel: «El parque jardín de un gran Sanatorio moderno destinado a las curas de reposo y a las enfermedades nerviosas" (Muñoz Seca, García Velloso 1928: 5) Tout est dit à demi-mot, comme feutré dans l'euphémisme pour ne pas parler concrètement de folie.

La didascalie n'est pas seulement climatique, elle commente le hors-scène pour aller dans le sens de ce qui vient dêtre évoqué: «El establecimiento responde por su vasta extensión y sistema a los más avanzados adelantos psiquiátricos. OpenDoor» (Muñoz Seca, García Velloso 1928: 5). Le mot est lâché, il s'agit bien d'un espace psychiatrique. Cependant l'imprécision reste de mise et la suite de la didascalie évoque un espace visuellement accueillant pour les malades logés dans des pavillons «separados unos de otros por bellos parques y jardines». L'espace psychiatrique en question tend donc à recréer l'habitat bourgeois de la villa de la famille aisée. Tout cela est confirmé par la suite de la didascalie qui indique que le pavillon de droite appartient au «matrimonio Fontillonga de Rencagua». Les trois pavillons sont dotés de "puertas y ventanas practicables» ce qui nous situe bien loin d'une folie enfermée. L'image de l'apaisement est construite on ne peut plus clairement: "Ambiente de Alegría y tranquilidad».

Le décalage se poursuit quand la didascalie commente l'action en présence. Luisa, infirmière, sert le thé à ses patients. Laction la caractérise comme servante dans un univers bourgeois: "trayendo la mesa bandeja en la que habrá un servicio de té y platos con manjares suculentos: pastas, dulces, frutas, tostadas, huevos... las tres [enfermeras] visten de blanco, con algún adorno de cabeza de la más refinada coquetería " (Muñoz Seca, García Velloso 1928: 5). Limage pavillonnaire a recréé dans l'enceinte de l'asile la structure sociale qui existe à l'extérieur. Ainsi, chaque patient a son infirmière particulière qui ressemble plus à une domestique quà une infirmière. Cet espace de l'anti-crise sur lequel souvre la pièce est bientôt confirmé par les paroles de l'infirmière Margarita qui affirme: "Aquí no se admiten locos. Esto en realidad no es más que un lujoso hotel científico » (Muñoz Seca, García Velloso 1928: 8). Dans cet espace de l’anticrise, on ne parle pas de fou, d’aliéné mais de neurasthénique, pas de folie mais de surmenage, pas d'enfermement mais de cure de repos et enfin, pas d'asile mais de luxueux hôtel scientifique.

«L'instant décisif» de la crise peut donc être construit spatialement par des espaces scéniques de l'anti-crise qui vont provoquer la rupture de l'inattendu quand cette dernière va y surgir sans crier gare. D’autres, comme Azorín construisent un espace théâtral évolutif qui fait croître progressivement la tension dramatique.

Dans Dr. Death de 3 a 5, le hors-scène complète le tableau d'un espace théâtral conçu pour faire naître «l'instant décisif». La salle d'attente possède, à 
gauche, une fenêtre qui donne sur le jardin de la consultation du médecin. Il s'agit là d'un espace imaginaire évolutif qui mène progressivement à la crise, chaque description du jardin reflétant l'évolution croissante de l'intensité dramatique. Le jardin est d'abord une vue rassurante et apaisante "un jardín tan bello» (Azorín 1927 : 1064). La deuxième évocation du jardin précise le paysage et l'ambivalence qu’il comporte: "¡Cómo me atrae este jardín!¡Ah, qué raro! Antes no había visto las siemprevivas; todo está lleno de siemprevivas... No sé qué pensar ¡Y esos cipreses tan altos, tan rígidos, tan negros! ¡Todo esto es un poco extraño!» (Azorín 1927: 1064). La troisième allusion au jardin est une vision funèbre: il est devenu cimetière: "¡Qué horror! ¡Horrible, horrible! ¡Socorro! ¡Auxilio! ¡Dios mío, Dios mío! Todo en el jardín está lleno de cruces, de tumbas; no se ven más que sepulturas.» Ce jardin hors-scène est donc un espace mouvant qui évolue au rythme des états d'âme du personnage qui le conduisent à la crise de panique. Le hors-scène est ici l'intériorité projetée en hallucination. Cet espace imaginaire cauchemardesque est également le marqueur d'un temps qui file vers la crise: «Y va pasando el tiempo. La tarde avanza. Llega el crepúsculo. Sí, la luz va decreciendo. (Va menguando la luz)» (Azorín 1927: 1068). L’espace devient angoissant sous la perte des repères spatio-temporels. La seule marque du temps qui passe est celle du temps cyclique, et bien sûr hautement symbolique, du jour qui décline et s'achemine vers sa fin.

La crise dans le théâtre freudien dépasse donc le simple motif de l'homme en crise et se situe au niveau d'une esthétique de la crise, d'une structure critique comprise à l'échelle du drame dans sa totalité. Revenons à l'étymologie grecque du terme de Krisis, à ce "jugement subjectif émis au terme de délibérations approfondies». La «mise en crise» pointée du doigt par Sylvain Diaz dans les dramaturgies européennes $\mathrm{du} \mathrm{XIX}^{\mathrm{e}}$ et $\mathrm{XX}^{\mathrm{e}}$ siècle, revient à penser le drame comme une crise, c'est-à-dire comme l'endroit de la représentation esthétique d'un jugement, d'une interrogation. C'est à partir de cette étymologie qu'il explicite l'appréhension contemporaine de la tragédie antique puis classique comme des crises. La tragédie est: «cette forme artistique unique dans l'Antiquité qui s'interroge sur la relation que l'agent entretient avec son action. Elle propose, de manière singulière, une opération critique relative à l'action humaine. Assurément, la tragédie grecque est mise en crise» (Diaz 2009: 52). La "mise en crise» se joue au niveau esthétique dans la tragédie antique, le cœur étant cet élément interrogateur qui "met en crise», propose le débat.

Le théâtre freudien qui nous intéresse se prête particulièrement à cette «mise en crise», en tant que représentation esthétique d'un choix et de ses conséquences. Il s'appréhende comme un théâtre réflexif, un théâtre de la mise en question, de la mise en débat esthétique.

Ce théâtre questionne le traitement social du fou à un moment où ce dernier est appréhendé à la ville et à la scène comme un malade mental. Des pièces comme Sinrazón ou Tararí interrogent esthétiquement la viabilité et la légitimité d'une méthode thérapeutique qui est à l’origine du drame par sa rigidité. La 
psychanalyse dans toute sa dimension mécanique et prescriptive est interrogée, sondée sur son bien-fondé.

Les deux pièces Tararí et Sinrazón mettent en place le débat au niveau esthétique en jouant sur la dialectique entre ordre et désordre. Dans les deux pièces, les internés, représentants d'un désordre, que la société a enfermés pour les empêcher de nuire, prennent le dessus dans l'asile sur les détenteurs de la raison. Le débat glisse dans un supposé désordre qui se donne à voir sur scène comme un ordre aussi bien structuré et harmonieux avant qu'après la révolte.

Dans Sinrazón, la fin du premier acte scelle une promesse entre Ballina et Don Manuel qui souhaite employer sa fortune à la construction d'un nouvel asile où le terme de fou serait à bannir. Le deuxième acte donne vie au projet de Don Manuel et le «Palacio de la Reina Beatriz» permet à ses hôtes de vivre dans l'illusion en leur donnant les moyens d'être ce qu'ils se figurent être. La reine Beatriz apparaît avec sa cour, vêtue d'un manteau d'hermine et d'une véritable couronne royale... Cette plongée dans le rêve de Don Manuel donne à voir le microcosme des internés, comme un tout harmonieux, comme un ordre que la psychanalyse, en la personne du docteur Ballina, va ébranler et mettre à mal en s’efforçant à rétablir la vérité objective et rationnelle de l'asile.

Une même esthétique de la continuité là où l'on attend une rupture vient dans Tararí confirmer cette «mise en crise esthétique» du traitement médical des fous. Dans cette pièce, l'avant et l'après révolte des internés construit une continuité, basée sur des jeux de parallélismes et d'imitation, qui interroge l'internement des fous par les détenteurs de la raison. L'attendu désordre sétablit comme un tout harmonieux sur fond d’organisation militaire dirigée par Don Paco. Le dernier tableau qui clôt la pièce met en scène un chaos visuel dans lequel anciens et nouveaux se confondent, pointant ainsi du doigt la dialectique qui oppose les fous aux hommes raisonnables comme un prétexte à la domination de l'homme par l'homme.

Ce théâtre réflexif propose donc d'interroger esthétiquement l'exclusion sociale que fonde la mise à lécart de l'asile. La décision, la «mise en crise» est celle du choix de la gestion des fous de leur propre destin et des conséquences de ce choix.

Dans Trance de Rivas Cherif, le débat sur les avancées freudiennes du psychisme se construit aussi au niveau esthétique. La séance d'hypnose mise en scène dans l'obscurité interroge le choix qui est fait d'aller sonder les recoins obscurs de lâme humaine. La pièce expose les conséquences de ce choix, de cette «investigación sicológica» (Rivas Cherif 1923:3). Lobscurité dit symboliquement l'intime et la transcription plastique des strates de la personnalité situées sous les couches de la conscience. Le drame de l'assassinat du médecin à la fin de la pièce interroge esthétiquement l'incursion du médecin dans l'intimité. Lassassinat est ainsi la cause finale d'une violation de l'espace intime que la scène dit à travers ce choix dramaturgique de l'obscurité. 
Au terme de ces réflexions, il apparaît que la figure du fou, devenu le malade dans ce théâtre freudien, incarne un théâtre « de la crise» en reprenant les critères définitionnels retenus par Sylvain Diaz. La peinture des dérèglements du Moi et du Monde construit un sujet critique sur lequel vient se greffer une structure critique qui s'appuie tantôt sur l'élaboration d'espaces de la crise, tantôt sur une temporalité de la crise. Enfin, ce théâtre, au-delà de son sujet et de sa structure, propose une "mise en crise», un questionnement sur les réactions de l'homme malade et sur le bien-fondé des avancées thérapeutiques de la psychanalyse.

\section{Bibliographie}

Andrés Álvarez, V., 1929, Tararí, Madrid, Revista de Occidente.

Arniches, C., 1926, La locura de don Juan: Tragedia grotesca en tres actos, Madrid, Prensa Moderna, «El Teatro» ${ }^{\circ} 23,1926$.

Diaz, S., 2009, Poétiques de la crise dans les dramaturgies européennes des $\mathrm{XX}^{e}$ et $X X I^{e}$ siècles, Lyon, Université Lumière Lyon 2.

Muñoz Seca, P., García Velloso, E., 1928, La cura: tragedia humorística en tres actos, Madrid, Rivadeneyra, «La Farsa " $n^{\circ} 39$.

Rivas Cherif, C. de, Trance, copie du manuscrit de la pièce jouée dans le cadre du Mirlo Blanco (1926-1927), prêtée par Begoña Riesgo avec l'autorisation de Enrique de Rivas Cherif, archives familiales.

Sánchez Mejías, I., 1988, Teatro, Gallego Morell, Antonio, Madrid, Espasa-Calpe, «Austral». 